\title{
COMUNIDADES DE LEPIDÓPTEROS NOTURNOS DESFOLHADORES DE EUCALIPTO EM TRÊS REGIÕES DO CERRADO DE MINAS GERAIS
}

\author{
Marcilio Fagundes ${ }^{1}$ \\ José Cola Zanuncio ${ }^{2}$ \\ Frederico Santos Lopes ${ }^{3}$ \\ Paulo De Marco Júnior ${ }^{4}$
}

\begin{abstract}
A diversified Lepidoptera fauna is found associated with Eucalyptus plantations in Brazil. From October 1990 to September 1991 twelve light traps were used in three regions of the Cerrado area with Eucalyptus plantations (Bom Despacho - BD, Três Marias - TM and Montes Claros - MC), in Minas Gerais State, Brazil. Twenty three Lepidoptera defoliator species were found in those regions. These species were affected by many factors, specially the plantation stage, the neighboring plant species, the type of brush vegetation and the species of Eucalyptus planted in a specific area. The samples were not significantly different between regions in species richness ( 12 to 17 species; $\mathrm{p}=0.065)$ or eveness $(0.53$ to $0.70 ; \mathrm{p}=0.779)$ in a randomized ANOVA test. Assuming a relation between the diversity and the vulnerability to pest outbreaks, it is sugested that the sampling points are equally vunerable. A cluster analysis revealed a clear separation between regions based in their species abundance and composition. In each region it is possible to show subsets of samples formed by the Eucalyptus species (MC) or the effect of the nearby regeneration areas (TM). These differences suggest a species substitution process that do not alter the fixed community structure shared by each region.
\end{abstract}

KEY WORDS. Lepidoptera, Eucalyptus, faunistic diversity

Os ecossistemas florestais suportam uma fauna diversificada de herbívoros, usualmente inconspícuos até que uma ou mais populações apresentem crescimento descontrolado, causando injúrias significativas às árvores. $\mathrm{O}$ impacto da herbivoria é função do tamanho ou biomassa das populações de herbívoros, as quais são influenciadas por fatores que afetam a natalidade, mortalidade e dispersão de tais populações (SCHOWALTER et al. 1986).

Explicações para as variações nas densidades de populações naturais de herbívoros devem ser buscadas nas interações dos vários fatores bióticos e abióticos que atuam sobre elas. Dentre estes, os mais comuns parecem ser a temperatura, a

1) Universidade Estadual de Montes Claros. $39400-000$ Montes Claros, Minas Gerais, Brasil.

2) Departamento de Biologia Animal, Universidade Federal de Viçosa. 36571-000 Viçosa, Minas Gerais, Brasil.

3) Universidade do Rio de Janeiro (UNIRIO). Rio de Janeiro, Rio de Janeiro, Brasil.

4) Departamento de Biologia Geral, Universidade Federal de Viçosa. 36571-000 Viçosa, Minas Gerais, Brasil. Endereço para correspondências. 
precipitação, o vento, as defesas químicas e físicas das plantas, as condições nutricionais das plantas, os inimigos naturais e as propriedades das comunidades de plantas (GRAHAN 1963; KNIGHT 196": SCHOWALTER et al. 1986).

No Brasil, os plantios homogêneos, principalmente do gênero Eucalyptus, representam ecossistemas passíveis de serem colonizados por várias espécies de insetos nativos. Estes insetos, representados principalmente pelas formigas cortadeiras (ZANUNCIO et al. 1992), coleópteros (ZANUNCIO et al. 1993) e lepidópteros (ZANUNCIO 1993), constituem importantes agentes depredadores que podem comprometer o rendimento da eucaliptocultura em todas as suas fases.

Em programas de manejo de pragas em florestas, os fatores ou processos que determinam a periodicidade e a amplitude das variações das populações de insetos são particularmente importantes (WATERS \& STARK 1980). Desta forma, procurouse através da análise de índices faunísticos, avaliar o efeito de alguns fatores ambientais sobre as populações dos principais lepidópteros desfolhadores de eucalipto, em três regiões do Estado de Minas Gerais.

\section{MATERIAL E MÉTODOS}

As populações dos lepidópteros desfolhadores de eucalipto foram estudadas por meio de amostragens periódicas da entomofauna associada a povoamentos de Eucalyptus spp. nas regiões de Bom Despacho, Três Marias e Montes Claros, Estado de Minas Gerais. Estas amostragens foram realizadas com armadilhas luminosas Intral AL 012, com lâmpadas negras, colocadas no campo por uma noite em intervalos quinzenais de outubro de 1990 a setembro de 1991. Em cada região foram instaladas quatro armadilhas que constituem pontos individuais de amostragem (Tab. I).

Tabela I. Carcterização dos locais amostrados nas três comunidades florestais estudadas. Legenda: (P) pastagens, (E) plantio de eucalipto, (B) brejos, $(A)$ açude, $(\mathbf{R})$ reservas nativas, (SU) subtropical úmido, (SUS) subtropical subúmido, (TSS) tropical seco, (LVEA) latossolo vermelho escuro argiloso, (LVED) latossolo vermelho escuro distrófico, (TRE) terra roxa estruturada, (Sp 1) Eucalyptus grandis, (Sp 2) E. urophyla, (Sp 3) E. cloeziana.

\begin{tabular}{|c|c|c|c|c|c|c|c|c|c|c|c|c|}
\hline \multirow[b]{2}{*}{ Amostras } & \multicolumn{4}{|c|}{ Bom Despacho } & \multicolumn{4}{|c|}{ Três Marias } & \multicolumn{4}{|c|}{ Montes Claros } \\
\hline & $1 \mathrm{~A}$ & $2 A$ & $3 A$ & $4 A$ & 1B & $2 \mathrm{~B}$ & $3 B$ & $4 B$ & $1 \mathrm{C}$ & $2 \mathrm{C}$ & $3 C$ & $4 \mathrm{C}$ \\
\hline Latitude (S) & $19^{\circ} 44^{\prime}$ & $19^{\circ} 44^{\prime}$ & $19^{\circ} 44^{\prime}$ & $19^{\circ} 44^{\prime}$ & $18^{\circ} 20^{\prime}$ & $18^{\circ} 20^{\prime}$ & $18^{\circ} 20^{\prime}$ & $18^{\circ} 20^{\prime}$ & $16^{\circ} 39^{\prime}$ & $16^{\circ} 39^{\prime}$ & $16^{\circ} 39^{\prime}$ & $16^{\circ} 39^{\prime}$ \\
\hline Longitude (W) & $44^{\circ} 15^{\prime}$ & $44^{\circ} 15^{\prime}$ & $44^{\circ} 15^{\prime}$ & $44^{\circ} 15^{\prime}$ & $45^{\circ}$ & $45^{\circ}$ & $45^{\circ}$ & $45^{\circ}$ & $43^{\circ} 39^{\prime}$ & $43^{\circ} 39^{\prime}$ & $43^{\circ} 39^{\prime}$ & $43^{\circ} 39^{\prime}$ \\
\hline Altitude (m) & 720 & 737 & 740 & 715 & 740 & 740 & 740 & 740 & 790 & 799 & 790 & 794 \\
\hline Espécie & Sp 1 & Sp 1 & Sp 1 & Sp 1 & Sp 2 & Sp 2 & Sp 2 & $\operatorname{Sp} 2$ & Sp 2 & $\mathrm{Sp} 3$ & Sp 3 & Sp 2 \\
\hline Plantio & $10 / 82$ & $10 / 82$ & $08 / 83$ & $10 / 83$ & $10 / 88$ & $10 / 88$ & $10 / 88$ & $10 / 88$ & $10 / 84$ & $11 / 84$ & $11 / 84$ & $11 / 84$ \\
\hline Espaçamento $(\mathrm{m}) 2$ & $2,0 \times 1,0$ & $2,0 \times 1,0$ & $2,0 \times 1,0$ & $2,0 \times 1,0$ & $2,0 \times 1,0$ & $2,0 \times 1,0$ & $2,0 \times 1,0$ & $2,0 \times 1,0$ & $3,0 \times 1,5$ & $3,0 \times 1,5$ & $3,0 \times 1,5$ & $3,0 \times 1,5$ \\
\hline Sub-bosque & Denso & ralo & ausente & ralo & ralo & ralo & ralo & ralo & ralo & ralo & ralo & ausente \\
\hline Divisas & $P+E$ & $P+E$ & $P+E$ & $\begin{array}{l}B+A+ \\
P+E\end{array}$ & $E$ & $E$ & $R+E$ & $R+E$ & $\mathrm{R}+\mathrm{E}$ & $E$ & $R+E$ & $E$ \\
\hline Clima & SU & SU & SU & su & sus & sus & sus & sus & TSS & TSS & TSS & TSS \\
\hline Solo & LVEA & LVEA & LVEA & LVEA & TRE & TRE & TRE & TRE & LVED & LVED & LVED & LVED \\
\hline
\end{tabular}

Os insetos capturados foram acondicionados em mantas entomológicas e enviados para o insetário da Universidade Federal de Viçosa, onde foram triados, 
quantificados e catalogados. Dos lepidópteros coletados, apenas os pertencentes à guilda de desfolhadores de eucalipto será aqui analisada. Informações sobre aspectos bionômicos destas espécies podem ser obtidas a partir de ZANUNCIO (1993).

A caracterização da fauna de lepidópteros das comunidades foi feita por meio da análise de índices de diversidade (HILl 1973) e equitatividade (AlATALO 1981). Testes para diferenças entre regiões foram feitos através de análise de variância (ANOVA) por testes de randomização, de acordo com MANLY (1991). O uso de modelos de randomização nestes testes têm as qualidades de não depender de uma distribuição particular dos dados e o cálculo direto das probabilidades sem o uso de funções auxiliares. As comunidades foram agrupadas usando-se o procedimento UPGMA (agrupamento pela média, sem pesos) sobre a matriz de dissimilaridades entre pontos amostrais, de acordo com LUDWIG \& REYNOLDS (1988). Como medida de dissimilaridade usou-se a distância absoluta relativa calculada a partir da matriz básica de abundância de espécies.

\section{RESULTADOS E DISCUSSÃO}

\section{Levantamento da fauna}

Foram coletados, nos 12 pontos amostrais, 34678 indivíduos pertencentes a 23 espécies de Lepidoptera consideradas importantes para a eucaliptocultura nacional. Dentre estas, Eupseudosoma aberrans (24,96\% dos indivíduos coletados), Eupseudosoma involuta (2,91\%), Psorocampa denticulata (3,04), Stenalcidia grosica $(51,29 \%)$ e Thyrinteina arnobia $(6,22 \%)$ foram as mais abundantes (Tab. II).

Observou-se correlação positiva entre a abundância média e a frequência das espécies ou seja, aquelas espécies que ocorreram em maior número de locais registraram densidades populacionais mais altas $\left(\mathrm{r}^{2}=0.528 ; \mathrm{F}=23.477, \mathrm{p}<0.001\right.$, figura 1). Esta relação pode decorrer de que espécies mais generalistas, dentro de um conjunto de espécies similares ecologicamente, devem apresentar distribuição mais ampla (BRown 1984). Espécies de Lepidoptera que possuem limites de tolerância mais largos, podem expandir mais rapidamente sua distribuição, resguardada as habilidades de dispersão de cada uma delas.

Por outro lado, supondo que as espécies de Lepidoptera que atacam atualmente o eucalipto tem como hospedeiros plantas filogeneticamente próximas a este (o que talvez as levasse a ser também quimicamente parecidas), lepidópteros mais generalistas quanto aos tipos de folhas poderiam ter maior sucesso em atacar o eucalipto. Desta forma também seria esperado uma maior densidade destas espécies nos cultivos de eucalipto e talvez uma dispersão mais rápida entre cultivos, alargando sua distribuição geográfica. Este mecanismo também está de acordo com as explicações de BROWN (1984), mas estende o foco de atenção para as relações entre plantas nativas hospedeiras e lepidópteros desfolhadores de eucalipto na busca de causas da ampla distribuição dos mesmos.

Dentro de uma área geográfica, a distribuição de cada espécie é irregular em diferentes graus devido à variação espacial das características físicas, a disponibilidade de recursos, e outras espécies que agem como competidoras, parasitóides ou 
predadoras. Muitas espécies, particularmente aquelas que possuem necessidades específicas, são formadas de algumas a muitas populações pequenas que estão amplamente separadas. Estas populações são susceptiveis à extinção e sua persistência em um local particular depende do influxo de migrantes de outras populações (BROWN 1984; HANSKI 1991).

Tabela II. Abundância total das espécies de lepidópteros noturnos desfolhadores de eucalipto, coletados com armadilhas luminosas de outubro de 1990 a setembro de 1991.

\begin{tabular}{|c|c|c|c|c|c|c|c|c|c|c|c|c|c|}
\hline \multirow{2}{*}{ Espécies } & \multicolumn{4}{|c|}{ Bom Despacho } & \multicolumn{4}{|c|}{ Três Marias } & \multicolumn{4}{|c|}{ Montes Claros } & \multirow{2}{*}{ Total } \\
\hline & $1 \mathrm{~A}$ & $2 A$ & $3 A$ & $4 A$ & $1 \mathrm{~B}$ & $2 B$ & $3 B$ & $4 B$ & $1 \mathrm{C}$ & $2 \mathrm{C}$ & $3 \mathrm{C}$ & $4 \mathrm{C}$ & \\
\hline \multicolumn{14}{|l|}{ ARCTIIDE } \\
\hline Eupseudosoma aberrans Schaus, 1905 & 1 & 2 & 4 & 9 & 789 & 1640 & 3432 & 1672 & 10 & 16 & 26 & 13 & 7614 \\
\hline Eupseudosoma involuta (Sepp, 1855) & 131 & 25 & 26 & 33 & 71 & 101 & 344 & 69 & 11 & 14 & 29 & 1 & 855 \\
\hline Idalus affinis Rotschild, 1910 & 0 & 0 & 0 & 0 & 0 & 0 & 0 & 0 & 0 & 1 & 0 & 00 & 1 \\
\hline Idalus sp. & 3 & 2 & 2 & 20 & 7 & 19 & 19 & 2 & 0 & 0 & 0 & 0 & 66 \\
\hline Lepidokirbyia vittipes (Walker, 1855) & 89 & 37 & 12 & 62 & 10 & 11 & 11 & 11 & 113 & 217 & 90 & 16 & 673 \\
\hline \multicolumn{14}{|l|}{ EUPTEROTIDAE } \\
\hline \multicolumn{14}{|l|}{ GEOMETRIDAE } \\
\hline Glena sp. & 0 & 0 & 0 & 0 & 130 & 249 & 237 & 34 & 11 & 23 & 11 & 15 & 710 \\
\hline Oxydia vesulia (Cramer, [1779]) & 27 & 220 & 40 & 131 & 0 & 0 & 0 & 0 & 20 & 21 & 8 & 3 & 470 \\
\hline Sabulodes caberata Guenéé, 1857 & 0 & 0 & 0 & 1 & 0 & 0 & 0 & 0 & 0 & 0 & 0 & 0 & 1 \\
\hline Stenalcidia sp. & 107 & 253 & 105 & 500 & 2619 & 6450 & 2228 & 1390 & 37 & 54 & 45 & 23 & 14111 \\
\hline Thyrinteina arnobia (Stoll, 1782) & 22 & 146 & 26 & 25 & 614 & 896 & 315 & 202 & 0 & 0 & 0 & 0 & 2246 \\
\hline Thyrinteina leucoceraea (Rindge, 1961) & 0 & 0 & 0 & 0 & 0 & 0 & 0 & 0 & 61 & 25 & 17 & 8 & 111 \\
\hline \multicolumn{14}{|l|}{ LYMANTRIIDAE } \\
\hline $\begin{array}{l}\text { Sarsina violascens (Henrrich-Schaeffer, } \\
1856 \text { ) }\end{array}$ & 21 & 37 & 31 & 26 & 178 & 112 & 247 & 87 & 62 & 38 & 22 & 16 & 877 \\
\hline \multicolumn{14}{|l|}{ NOTODONTIDAE } \\
\hline Blera sp. 1 & 1 & 0 & 1 & 0 & 2 & 17 & 6 & 3 & 0 & 2 & 0 & 0 & 32 \\
\hline Blera sp. 2 & 12 & 5 & 6 & 4 & 0 & 0 & 0 & 0 & 139 & 43 & 5 & 49 & 263 \\
\hline Nystalea nyseus (Cramer, [1775]) & 0 & 1 & 1 & 0 & 2 & 10 & 35 & 3 & 1 & 0 & 0 & 0 & 53 \\
\hline Psorocampa denticulata (Schaus, 1901) & 17 & 57 & 17 & 5 & 62 & 345 & 322 & 123 & 64 & 21 & 27 & 9 & 1069 \\
\hline \multicolumn{14}{|l|}{ SATURNIIDAE } \\
\hline Citheronia laocoon (Cramer, [1777]) & 0 & 0 & 0 & 0 & 10 & 17 & 1 & 2 & 1 & 0 & 0 & 0 & 31 \\
\hline Eacleas imperialis magnifica (Walker, 1855) & 1 & 2 & 0 & 2 & 0 & 0 & 0 & 0 & 4 & 0 & 2 & 0 & 11 \\
\hline Automeris illustris (Walker, 1855) & 8 & 4 & 2 & 1 & 0 & 1 & 0 & 0 & 7 & 6 & 0 & 3 & 32 \\
\hline Dirphia rosacordis (Walker, 1856) & 10 & 15 & 53 & 41 & 1 & 0 & 0 & 0 & 232 & 40 & 32 & 81 & 505 \\
\hline Hyperchiria incisa (Walker, 1855) & 6 & 2 & 1 & 1 & 0 & 3 & 1 & 0 & 6 & 4 & 9 & 8 & 41 \\
\hline Lonomia sp. & 0 & 0 & 3 & 0 & 0 & 0 & 0 & 0 & 7 & 4 & 8 & 2 & 24 \\
\hline Total & 476 & 821 & 361 & 895 & 4795 & 9857 & 7198 & 3090 & 786 & 529 & 331 & 247 & 29894 \\
\hline
\end{tabular}

Normalmente, espécies que utilizam diferentes recursos podem atingir populações elevadas, colonizando vários outros sítios em uma área relativamente grande. Por outro lado, espécies que utilizam recursos restritos são pouco abundantes, restringindo sua distribuição a locais específicos numa região geográfica (BROWN 1984).

Os fitófagos estudados constituem um grupo de insetos nativos que se encontram em fase inicial de convivência adaptativa com os cultivos de eucalipto (ZANUNCIO et al. 1990). Neste novo ecossistema, estes insetos utilizam, além do eucalipto, vários outros recursos oferecidos pelo sub-bosque. Esta variedade de recursos passível de ser utilizada, constitui, provavelmente, um dos fatores responsáveis pela ampla distribuição geográfica destes desfolhadores, conforme mostram 
os trabalhos de PEREIRA (1992) e BARROS (1984). Estas características (herbívoros generalistas e ampla distribuição geográfica) associadas a fatores ecológicos locais podem ajudar a compreender as causas das explosões demográficas irregulares no tempo e no espaço deste grupo de lepidópteros (ZANUNCIO 1993).

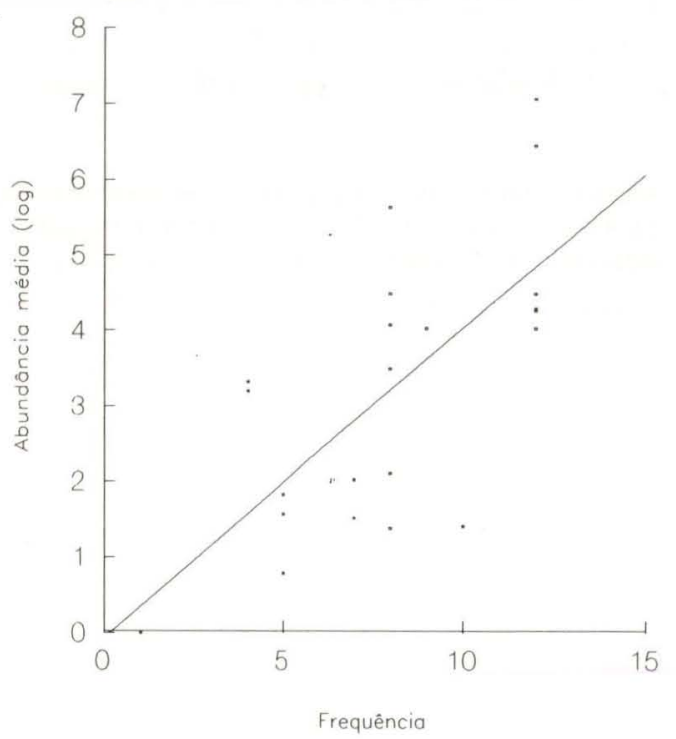

Fig. 1. Relação entre abundância média e frequência de ocorrência para as espécies coletadas.

\section{Caracterização das comunidades}

De modo geral, os pontos amostrais de uma mesma região não apresentaram diferenças acentuadas nos índices de diversidade. Contudo, as amostras de Três Marias apresentaram índices relativamente menores que os das demais regiões (Tab. III). Nesta região os plantios de eucalipto encontravam-se em estado sucessional menos avançado (Tab. I), o que poderia justificar a ocorrência de espécies dominantes e, consequentemente, a menor diversidade observada. De fato, a maioria dos surtos de lepidópteros em eucalipto têm ocorrido em plantios de dois a cinco anos de idade (ZANUNCIO 1993).

Outro fator que deve ser considerado é a quantidade de luz que atravessa o extrato arbóreo superior, formado pelos eucaliptos, e atinge o sub-bosque. Segundo CALEGARIO et al. (1993) a maior incidência de luz favorece o desenvolvimento do sub-bosque, resultando em uma maior diversidade florística. Estes autores tambem salientam que a espécie de eucalipto cultivada afeta a diversidade florística devido a diferenças de cobertura.

Embora os índices de diversidade tenham sido menores na região de Três Marias, onde foram amostradas áreas com Eucalypus urophylla, não se pode relacionar consistentemente esta diferença de estrutura de comunidade com a espécie cultivada. Como exemplo cita-se, em Montes Claros, as áreas de E. 
urophylla que mostram estrutura de comunidades largamente sobreposta à de $E$. cloeziana.

Os testes de randomização não mostraram diferenças significativas entre as regiões para nenhum dos parâmetros de estrutura de comunidade analisados (Tab. III). Isto sugere a existência de uma estrutura geral compartilhada por diferentes comunidades de lepidópteros desfolhadores de eucalipto, a despeito da espécie cultivada e, sendo modificada pela heterogeneidade do plantio e outras variáveis ambientais.

Tabela III. Diversidade e equitabilidade de lepidópteros desfolhadores nas três áreas amostradas. (NO) Riqueza em espécies, (N1) número de espécies abundantes, (N2) número de espécies muito abundantes e (E) equitatividade segundo HILL (1973).

\begin{tabular}{lccccc}
\hline Regiöes & Amostras & NO & N1 & N2 & $E$ \\
\hline \multirow{3}{*}{ Bom Despacho } & 1 & 16 & 7,90 & 5,83 & 0,70 \\
& 2 & 16 & 6,40 & 4,81 & 0,70 \\
& 3 & 16 & 4,86 & 2,90 & 0,48 \\
Très Marias & 4 & 14 & 6,74 & 4,24 & 0,56 \\
& 1 & 14 & 3,14 & 2,14 & 0,53 \\
& 2 & 13 & 4,16 & 3,01 & 0,64 \\
Montes Claros & 3 & 12 & 3,49 & 1,70 & 0,68 \\
& 4 & 13 & 2,81 & & 0,68 \\
ANOVA * & 1 & 17 & 8,38 & 6,23 & 0,54 \\
& 2 & 16 & 8,09 & 5,02 & 0,70 \\
\end{tabular}

*. Nivel de significância atingido em teste por ANOVA randomizada para $\mathrm{HO}$ de igualdade entre as regiōes.

\section{Classificação das comunidades}

O padrão de agrupamento das 12 amostras encontra-se ilustrado no dendrograma da figura 2. Este mesmo padrão, encontrado a partir da distância absoluta entre amostras, foi encontrado quando utilizou-se outras medidas, como porcentagem de dissimilaridade e distância euclidiana, sendo portanto considerado robusto.

As amostras de Bom Despacho e Três Marias foram mais similares entre si que com Montes Claros, apesar de Três Marias e Montes Claros apresentarem amostras com a mesma espécie de eucalipto (E. urophylla). Isto sugere que grandes padrões de similaridade são devidos a fatores climáticos e biogeográficos agindo sobre a composição de espécies de cada local. Três Marias e Bom Despacho, que estão mais ao sul e em menores altitudes (Tab. I), podem ser classificadas como clima tipo Cwa (ANTUNES 1980), enquanto Montes Claros apresenta clima Aw na classificação de Köppen.

Em Bom Despacho, a amostra 1A destacou-se, provavelmente, pelo fato de apresentar um sub-bosque mais desenvolvido (Tab. I) o que pode ser responsável pela diversidade relativamente alta observada neste local, conforme sugere PRICE (1984) ao mencionar que habitats mais complexos podem suportar um maior número de espécies de herbívoros. 


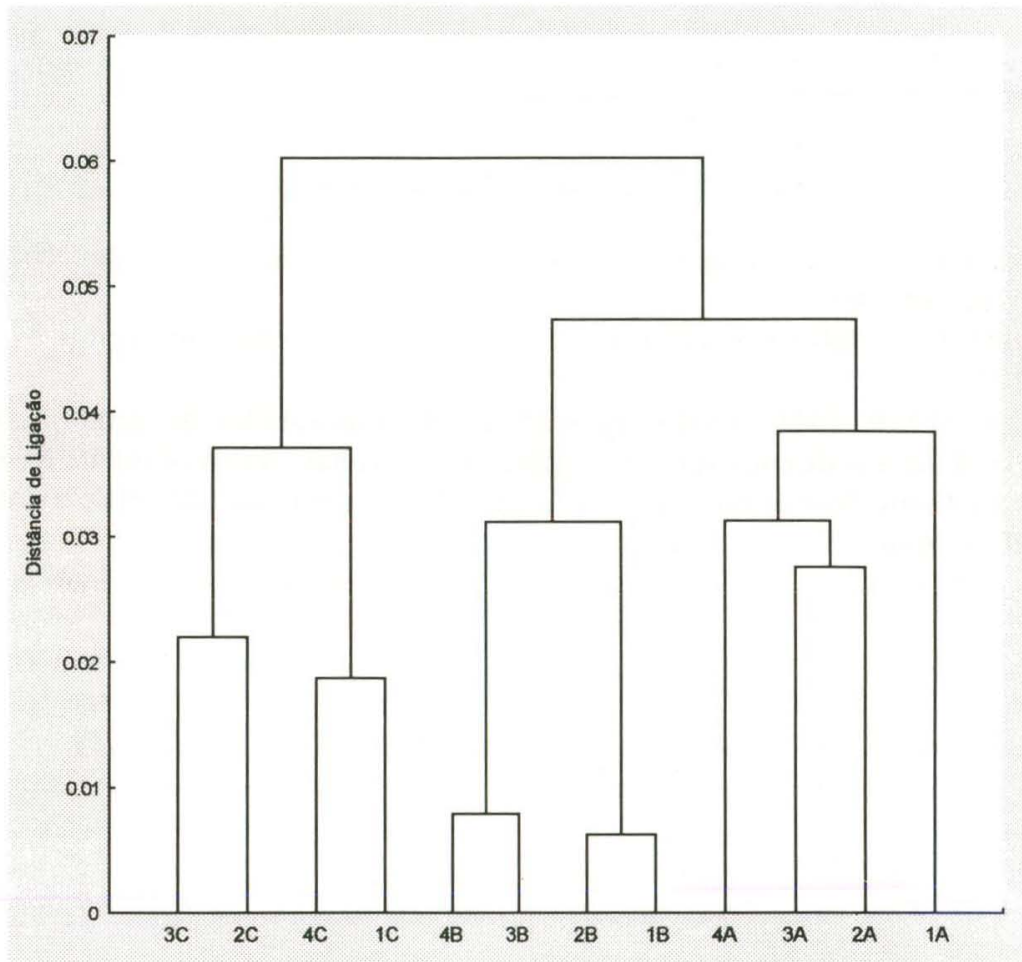

Fig. 2. Dendrograma da similaridade da fauna de lepidópteros entre os pontos amostrados.

Na região de Três Marias as amostras 1B e 2B formaram um grupo distinto de 3B e 4B. O único fator que diferencia estes subgrupos é a presença de reservas nativas ao redor dos pontos $3 \mathrm{~B}$ e $4 \mathrm{~B}$ (Tab. I). Estas matas residuais podem funcionar como reservatório de inimigos naturais como predadores, parasitas e parasitóides, levando a um controle de espécies muito abundantes, mas também como fonte de lepidópteros para a colonização da área. Neste sentido, a dinâmica de espécies ocorrentes nas plantações de eucalipto pode assemelhar-se àquelas preditas pela teoria de equilíbrio de biogeografia de ilhas (MACARTHUR \& WILSON 1967), onde as matas residuais comportam-se como reservatório de espécies para a colonização dos talhões de eucalipto.

Em Montes Claros, as comunidades $2 \mathrm{C}$ e $3 \mathrm{C}$ formam um subgrupo distinto de $1 \mathrm{C}$ e $4 \mathrm{C}$. Estes dois subgrupos se diferenciam, principalmente, com relação à espécie de eucalipto plantada, respectivamente, E. cloeziana e E. urophylla. As folhas de eucalipto contêm compostos secundários como taninos e óleos essenciais que podem funcionar como defesas contra herbívoros (OHMART \& EDWARDS 1991). Entretanto, sabe-se que o gênero Eucalyptus é formado por centenas de espécies com características morfológicas e resistência a herbívoros. Por isso, estudos envolvendo resistência espécie-específica podem auxiliar os programas de manejo das pragas desta essência florestal. 
AGRADECIMENTOS. Este trabalho foi parcialmente financiado pelo CNPq, FAPEMIG, CAF

- Florestal, Pains Florestal, REFLORALJE e SIF.

\section{REFERÊNCIAS BIBLIOGRÁFICAS}

Alatalo, R.V. 1981. Problems in the measurement of evenness in ecology. Oikos 37 (2): 199-204.

ANTUNES, F.Z. 1980. Caracterização do cerrado de Minas Gerais. Inf. Agrop. 6(1): 52-63.

BARros, M.E.P. 1984. Análise faunística das comunidades de lepidópteros desfolhadores de eucalipto em regiões do Maranhão, Bahia, Espírito Santo e São Paulo. Tese de Mestrado, não publicada, Universidade Federal de Viçosa, Viçosa, 63p.

BROWN, J.H. 1984. On the relationship between abundance and distribution of species. Am. Nat. 124: 255-279.

Calegário, N.; A.L. Souza; L.C. Marangon \& A.F. Silva. 1993. Parâmetros florísticos e fitossociológicos da regeneração de espécies arbóreas nativas no sub-bosque de povoamentos de Eucalyptus. Rev. Árvore 17 (1): 16-29.

GRAHAN, K. 1963. Concepts of forest entomology. New York, Reinold, 338p.

HANSKI, I. 1991. Single-species metapopulation dynamics: concepts, models and observations. Biol. J. Linn. Soc. 42: 17-38.

HiLl, M.O. 1973. Diversity and evenness: a unifying notation and its consequences. Ecology 54: 427-432.

KNIGHT, F.B. 1967. Principles of forest entomology. New York, McGraw-Hill, $461 \mathrm{p}$.

LudWIG, J.A. \& J.F. REYNOLDS. 1988. Statistical Ecology. A Primer on Methods and Computing. New York, John Wiley \& Sons, 336p.

MACARTHUR, R.H. \& E.O. WILSON. 1967. The Theory of Island Biogeography. Princeton, Princeton University Press, 203p.

MANLY, B.F.J. 1991. Randomization and Monte Carlo Methods in Biology. London, Chapman and Hall, $281 \mathrm{p}$.

OHMART, C.P. \& P.B. EDWARDS. 1991. Insect herbivory on Eucalyptus. Ann. Rev. Entomol. 36: 637-657.

PEREIRA, J.M.M. 1992. Fauna de lepidópteros-praga de eucalipto em regiões de Minas Gerais e São Paulo. Tese de Mestrado, Universidade Federal de Viçosa, Viçosa, 76p.

PRICE, P.W. 1984. Insect ecology. New York, John Wiley, 607p.

Schowalter, T.D.; W.W. Hargrove \& D.A. Crossley JR. 1986. Herbivory in forested ecosystems. Ann. Rev. Entomol. 31: 177-196.

WATERS, W.E. \& R.W. STARK. 1980. Forest pest management: concept and reality. Ann. Rev. Entomol. 25: 479-509.

ZANUNCIO, J.C. 1993. Lepidoptera desfolhadores de eucalipto: Biologia, ecologia e controle. Viçosa, IPEF/SIF, $141 \mathrm{p}$.

ZANUNCIO, J.C.; M. FAGUndes; N. ANJOS; T.V. ZANUnCIO \& L.R. CAPITANI. 1990. 
Levantamento e flutuação populacional de lepidópteros associados a eucaliptocultura: V - Região de Belo Oriente, Minas Gerais, junho de 1986 a maio de 1987. Rev. Árvore 14 (1): 35-44.

ZANUNCiO, J.C.; L. Couto; M. Fagundes \& T.V. ZANunCiO. 1992. Eficiência de isca granulado Mirex-s, à base de sulframida no controle da formiga cortadeira Atta laevigata (F. Smith, 1858). Rev. Árvore 16 (3): 357-361.

Zanuncio, J.C.; M.A.L. BRAGançA; A.J. LaRAnJeIro \& M. FAgundes. 1993. Coleópteros associados à eucaliptocultura em regiões de São Mateus e Aracruz, Espírito Santo. Rev. Ceres 40 (232): 583-589.

Recebido em 10.I.1995; aceito em 22.XI.1996. 